\title{
The effect of non-pharmacological interventions on physical restraint reduction in intensive care units: a protocol for an umbrella review of systematic reviews and meta-analysis
}

\author{
Nianqi Cui ${ }^{1}$, Dandan Chen ${ }^{2}$, Yuping Zhang ${ }^{1}$, Hui Zhang ${ }^{2}$, Xiaowei He ${ }^{3}$, Chuanqi Ding ${ }^{1,4}$, Jingfen Jin \\ ${ }^{1}$ The Second Affiliated Hospital Zhejiang University School of Medicine (SAHZU), Hangzhou, China; ${ }^{2}$ Faculty of Nursing, Zhejiang University \\ School of Medicine, Hangzhou, China; ${ }^{3}$ Library, Zhejiang University, Hangzhou, China; ${ }^{4}$ Changxing Branch Hospital of SAHZU, Huzhou, China \\ Correspondence to: Jingfen Jin, RN, MHA. Professor, Assistant Dean, Executive President, The Second Affiliated Hospital of Zhejiang University \\ School of Medicine (SAHZU), No. 88 Jiefang Road, Shangcheng District, Hangzhou 310009, China; Changxing Branch Hospital of SAHZU, No. \\ 66 Taihu middle Road, Changxing Country, Huzhou 313100, China. Email: zrjzkhl@zju.edu.cn.
}

\begin{abstract}
Background: As the last resort in intensive care units, physical restraint reduction is affected by various interventions. Several non-pharmacological interventions may directly reduce physical restraints, such as staff education, or indirectly reduce physical restraint, such as delirium prevention; however, their effectiveness has remained inconclusive. Therefore, we devised a protocol for umbrella reviews to summarize the evidence integrating data of different non-pharmacological interventions that may reduce physical restraint use.

Methods: The umbrella review will be conducted following the methodology formulated by the Joanna Briggs Institute (JBI). Electronic databases, including Web of Science, PubMed, EMBASE, PsycInfo, Psyc Articles, Psychology and Behavioral Science Collection, Cumulative Index to Nursing and Allied Health Literature (CINAHL), JBISRIS (JBI Database of Systematic Reviews and Implementation Reports), Cochrane Database of Systematic Reviews, China National Knowledge Infrastructure (CNKI, for Chinese literature), SinoMed (for Chinese literature), and WANFANG DATA (for Chinese literature), will be searched to identify articles published from January 2016 to December 2020. A systematic review and metaanalysis quality will be critically assessed by AMSTAR 2 (A Measurement Tool to Assess Systematic Reviews). According to the GRADE (Grades of Recommendation, Assessment, Development, and Evaluation) guidelines, the evidence quality of each intervention will be assessed. Overlapping studies and the excess significance test will be performed to assess whether previous evidences are bias.

Discussion: This protocol was devised according to the guidelines of the Preferred Reporting Items for Systematic Review and Meta-Analysis Protocols (PRISMA-P). Umbrella reviews will be an excellent supplement to the evidence of the guideline adaptation and provide a broader picture of non-pharmacological interventions that may reduce the use of physical restraint, which can provide critical care nurses in intensive care units with the evidence they need.
\end{abstract}

Trial Registration: This umbrella review protocol was documented in the PROSPERO registry (CRD42021242586).

Keywords: Physical restraints; non-pharmacological interventions; intensive care units; umbrella review

Submitted Mar 18, 2021. Accepted for publication May 02, 2021.

doi: 10.21037/apm-21-626

View this article at: https://dx.doi.org/10.21037/apm-21-626 


\section{Introduction}

Physical restraint $(\mathrm{PR})$ is defined as any action or procedure that prevents a person's free body movement to a position of choice and/or normal access to his/her body by the use of any method attached or adjacent to a person's body that he/she cannot control or remove easily (1). Benbenbishty and colleagues (2) revealed in their point prevalence article that investigated the application of PR in European countries, preventing self-extubation was the first major cause initiating PR, whereas preventing tube, line, or drain removal was the second reason in intensive care units (ICUs). Nonetheless, many articles report the continuous occurrence of line removal and self-extubation even though PRs are used, and the PR rate in unplanned extubation is reported to be $25-87 \%$ (3). PR application may adversely affect patients and lead to reactions such as fracture, damage to soft tissue, impaired cognition, delirium, agitation, withdrawal, fear, loss of dignity, and emotional flooding (4-6). Such experiences may also affect their families, and families who witness the patient with PR during visitation probably experience more familial distress (7). Therefore, certain regulations and policies have been released to reduce PR use across numerous nations (8). The Intensive Care Society's (ICS) guidelines regarding the use of PR state that the least restrictive and proportionate response should be chosen and utilized for the least amount of time possible. Restraint must never be used as an acceptable strategy for addressing a shortfall in staffing (9). However, PR is still used frequently. A recent multicenter cross-sectional study conducted in China showed that the use of PR in ICUs was $59.07 \%$ (10). Another study involving 68 ICUs in the United States showed that the rate of PR in ICUs was $33 \%(11)$.

The use of PR is affected by many factors, including nurses' knowledge, attitudes, perception, and decisionmaking ability. Therefore, providing staff education to increase the understanding of evidence-based best practices and alternatives to $\mathrm{PR}$ is considered a direct intervention to reduce PR use (12). Several interventions may indirectly reduce PR. Compared with patients with no mechanical ventilation (MV), the PR rate is higher in those who undergo MV $(2,13)$. Reducing the duration of MV may potentially reduce PR. Similar interventions are used for delirium prevention. Delirium is a rapid onset, reversible, fluctuating condition characterized by inattention, changes in cognition, disordered sleep-wake cycle, and increased or decreased psychomotor activity (14). Routine observation and active intervention are needed for the prevention or management of delirium (15). Prevention measures are usually classified as non-pharmacological or pharmacological. Clinically, pharmacological interventions are generally applied in treating ICU delirium, and longterm treatment is needed when its origin is determined (16). Furthermore, the sedative action of drugs can cause aspiration pneumonia or respiratory depression (16). The ICS guidance for the use of PR in UK adults in ICUs recommends that non-pharmacological means of preventing and treating delirium should be in place. This includes, but is not limited to, measures such as noise management, lights, sleep hygiene, and orientation (9).

Non-pharmacological interventions such as complete ABCDEF (assess, prevent, and manage pain; both spontaneous awakening and breathing trials; choice of analgesia and sedation; delirium assessment, prevention, and management; early mobility and exercise; family engagement and empowerment) bundle performance has been associated with a lower likelihood of delirium $(11,17)$. However, non-pharmacological interventions such as physical environment (private rooms with physical barriers between bed spaces and wall-to-wall exterior windows, facing the window and not the wall, single-bedroom with a dedicated toilet) have been unsatisfactory ( $95 \%$ confidence interval: $0.39,1.55, \mathrm{P}=0.469)$ (15). Non-pharmacological intervention such as early rehabilitation is effective in preventing ICU delirium in systematic reviews (SRs) (18); however, other SRs did not support this conclusion (19). Early rehabilitation is one of many candidates for nonpharmacological interventions for delirium prevention. The same situation also exists for many other nonpharmacological interventions, which may become potential interventions to reduce PR. More and more studies have been conducted to review PR-targeted non-pharmacological interventions systematically, and a suitable and logical next step is to conduct reviews of existing SRs. The umbrella review compares numerous treatments to metaanalysis (MA) and SRs that only compare one treatment. Additionally, it also compares results from different reviews and provides the evidence necessary for decision-making in healthcare.

The present study is part of a guideline adaptation study of PR (20). Based on CAN-IMPLEMENT @ (21), a method for guideline adaptation and implementation, evidence comes from guidelines and SRMAs (systematic reviews and meta-analysis). Therefore, the present umbrella review aimed to broadly review results from SRMAs for nonpharmacological interventions to reduce PR. We present 
the following article following the PRISMA-P reporting checklist (available at https://dx.doi.org/10.21037/apm-21626) $(22,23)$.

\section{Methods}

The methodology of the umbrella review will be formulated by the Joanna Briggs Institute (JBI) (24). This umbrella review is registered at PROSPERO (CRD42021242586).

\section{Search strategy and study selection}

Electronic databases, including Web of Science, PubMed, EMBASE, PsycInfo, Psyc Articles, Psychology and Behavioral Science Collection, Cumulative Index to Nursing and Allied Health Literature (CINAHL), JBISRIS (JBI Database of Systematic Reviews and Implementation Reports), Cochrane Database of Systematic Reviews, China National Knowledge Infrastructure (CNKI, for Chinese literature), SinoMed (for Chinese literature), and WANFANG DATA (for Chinese literature), will be searched to identify articles published from January 2016 to December 2020. We will only include SRMAs in the last 5 years because research syntheses carried out over the last 5-10 years can mirror the original/primary studies that were performed 30 years ago and enrolled in the located research syntheses (24). Additionally, we will manually search reference lists from the screened articles to prevent omitting any related article. The search terms are shown below:

* Populations (P): adult intensive care patients.

* Interventions (I): any potential non-pharmacological interventions that may reduce $\mathrm{PR}$, including but not limited to the management of $\mathrm{PR}$, unplanned extubation, mechanical ventilation, pain, agitation/ sedation, delirium, and visiting/family engagement in the ICU.

* Professionals (P): mainly targeted for critical care nurses.

- Outcomes (O): PR rate, unplanned extubation rate, $M V$ time, weaning time, reintubation rate, extubation failure, delirium incidence, delirium duration, pain severity.

- Healthcare (H): adult ICUs.

The example of search terms is provided in Table 1. After screening titles and abstracts, the full texts of potential studies will be obtained. The exclusion criteria are nonSRMAs, non-adult ICU, non-Chinese and English
SRMAs, SRMAs with pharmacological interventions only, SRMAs without relevant outcome indicators, SRMAs with incomplete or missing analysis data, and unable to contact the original author and SRMAs with repeated reports. Two authors (NC and DC) are responsible for selecting articles independently, and any disagreement between them will be resolved by the opinion of a third investigator (YZ).

\section{Data extraction}

The following data will be collected: detailed information (first author, year of publication), objectives, language, number of databases for searching, period of searching, types of included studies, characteristics of participants, control and intervention types, outcomes of interest, enrolled study and participant numbers, synthesis methodology, assessment of quality, combined effect size and $95 \%$ CIs of all outcomes, and assessment of heterogeneity. Figure 1 is the data extraction form. Data from articles enrolled in every SRMA will also be collected (including first author and year of publication) to construct the study-citation matrix among the available SRMAs. Lastly, we will also obtain observations of all outcomes, together with basic incidence/risk from the enrolled articles, to estimate the power of the test and conduct excess significance tests. Two reviewers (NC and DC) will collect data from the relevant publications independently, and any disagreement between them will be resolved by the supervisor's opinion $(\mathrm{JJ})$.

\section{Quality assessment}

SRMA quality will be critically assessed by AMSTAR 2 (A Measurement Tool to Assess Systematic Reviews) (25). Although AMSTAR 2 consists of 16 items, critical domains include items 2, 4, 7, 9, 11, 13, and 15. Two members (NC and DC) will use AMSTAR 2 (Chinese version) (26) to assess the quality of the included SRMAs. Any disagreement between them will be resolved by the opinion of a third investigator (YZ). Upon reaching a consensus among all researchers, SRMAs with more than half of the critical domains not satisfied will be eliminated.

We will assess the evidence quality of each intervention according to the GRADE (Grades of Recommendation, Assessment, Development, and Evaluation) guidelines (27). Specifically, evidence quality will be evaluated as very low, low, moderate, and high. A randomized study design indicates high evidence quality, whereas an observational 
Table 1 Example of search terms

Search strategy for Cochrane Database of systematic reviews

\#1 MeSH descriptor: [Restraint, Physical] explode all trees

\#2 ((physical or mechanical) near (constraint* or restraint $\left.\left.{ }^{\star}\right)\right)$ : ti,ab,kw

\#3 ((disallow* or limit* $^{*}$ or restrict* or stop*) near (movement*)): ti,ab,kw

\#4 ((ankle ${ }^{\star}$ or appendage ${ }^{\star}$ or arm ${ }^{\star}$ or body or bodies or foot or feet or hand ${ }^{\star}$ or leg $^{\star}$ or limb $^{\star}$ or patient* or wrist*) near (tie or bedrail $^{\star}$ or belt $^{\star}$ or strap ${ }^{\star}$ or mitten ${ }^{\star}$ or jacket*)): ti,ab,kw

\#5 MeSH descriptor: [Airway Extubation] explode all trees

\#6 MeSH descriptor: [Ventilators, Mechanical] explode all trees

\#7 MeSH descriptor: [Respiration, Artificial] explode all trees

\#8 ((unplanned or self or accidental or ventilat*) near (extubat* or reintubat*)): ti,ab,kw

\#9 ((mechanic ${ }^{\star}$ or artificial or positive-pressure or wean* or liberat*) near (ventilat*or respirat* or breathing)): ti,ab,kw

\#10 MeSH descriptor: [Aggression] explode all trees

\#11 MeSH descriptor: [Psychomotor Agitation] explode all trees

\#12 MeSH descriptor: [Conscious Sedation] explode all trees

\#13 MeSH descriptor: [Behavior Control] explode all trees

\#14 ((abusive or challenging or disturbed or disruptive or aggression or agonistic or restless* or anger or angry or assault or rage* or hostil* $^{*}$ or threat* or violen*) near $\left(\right.$ behav $\left.\left.^{\star}\right)\right)$ : ti,ab,kw

\#15 (daily sedation interruption or crisis intervention): ti,ab,kw

\#16 MeSH descriptor: [Delirium] explode all trees

\#17 MeSH descriptor: [Emergence Delirium] explode all trees

\#18 MeSH descriptor: [Neurocognitive Disorders] explode all trees

\#19 (delirious*): ti,ab,kw

\#20 ((abnormal ${ }^{\star}$ or change* or dysfunction* or decline or deterioration or diminish ${ }^{\star}$ or disorders or mental* or disturbed $\left.{ }^{\star}\right)$ near $\left(\right.$ attention $^{*}$ or cognitive or consciousness or perception or neurocognitive)): ti,ab,kw

\#21 ((brain syndrome or cerebral insufficiency) near (acute)): ti,ab,kw

\#22 MeSH descriptor: [Pain, Procedural] explode all trees

\#23 MeSH descriptor: [Pain, Postoperative] explode all trees

\#24 MeSH descriptor: [Pain Management] explode all trees

\#25 MeSH descriptor: [Pain Measurement] explode all trees

\#26 MeSH descriptor: [Analgesics, Non-Narcotic] explode all trees

\#27 ((procedural or postoperative or management or measurement) near (discomfort or ache*)): ti,ab,kw

\#28 MeSH descriptor: [Decision Making, Shared] explode all trees

\#29 MeSH descriptor: [Family Separation] explode all trees

\#30 ((open or flexible or restrictive or family) near (visitation or visiting)): ti,ab,kw

\#31 ((patient-cent?red or family-cent?red) near (care or nursing)): ti,ab,kw

\#32 ((shared or sharing) near (decision-making or decision making)): ti,ab,kw

\#33 (SDM): ti,ab,kw

Table 1 (continued) 
Table 1 (continued)

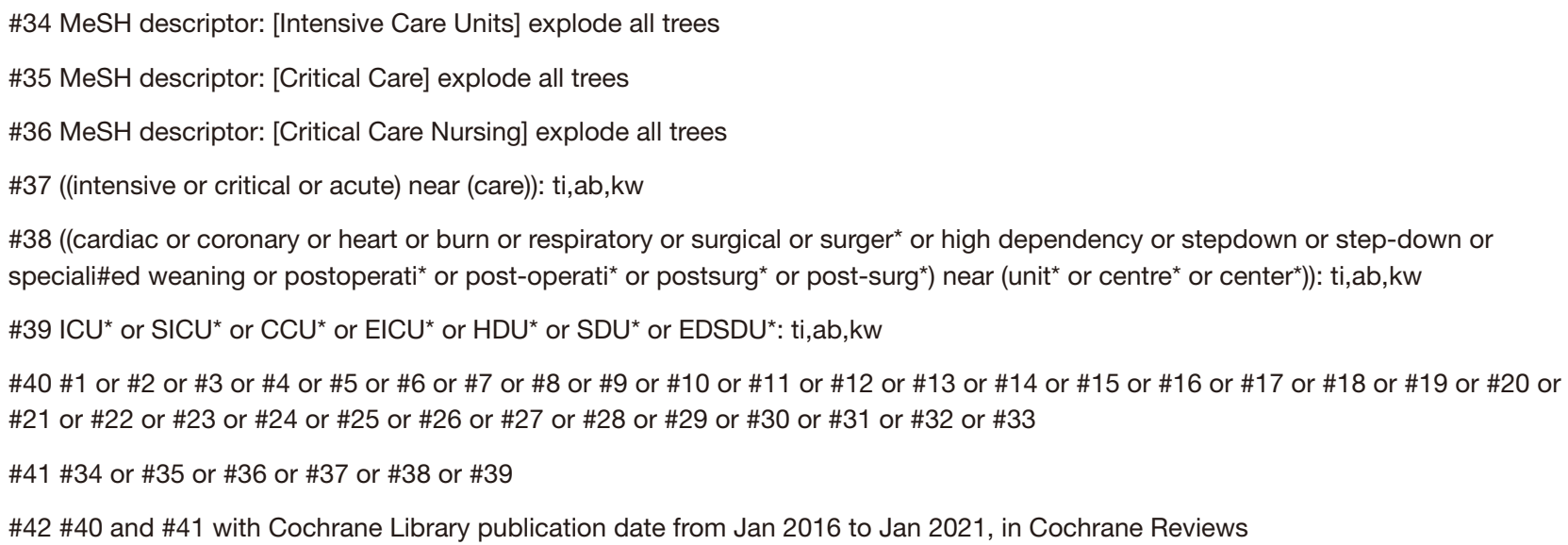

\begin{tabular}{|l|l|l|l|l|l|l|l|l|l|l|l|}
\hline Author/year & Language & Objectives & $\begin{array}{l}\text { Number of } \\
\text { databases }\end{array}$ & $\begin{array}{l}\text { Search } \\
\text { date }\end{array}$ & $\mathrm{N} / \mathrm{n}$ & $\begin{array}{l}\text { Types of } \\
\text { studies }\end{array}$ & Participants & $\begin{array}{l}\text { Intervention } \\
\text { vs. control }\end{array}$ & Outcome & $\begin{array}{l}\text { Quality } \\
\text { assessment }\end{array}$ & $\mathrm{I}^{2}$ \\
\hline & & & & & & & & & & \\
\hline
\end{tabular}

Figure 1 Data extraction forms. N, number of included studies; n, number of included participants.

design predicts poor evidence quality. The original articles will be obtained if needed. At a $<400$ sample size, imprecision is downgraded as recommended by the grade group. Also, $\mathrm{I}^{2}>50 \%$ indicates tremendous heterogeneity in point estimates for the interventional effect, so that inconsistency will be downgraded.

\section{Statistical analysis}

The study-citation matrix will be created to determine the intersected level among the enrolled SRMAs (28), where every column indicates a single SRMA (index review) while every row indicates a single study enrolled into the SRMAs (index publication), as shown in Figure 2. Then, we will predict the intersected level by covered area (CA) and the corrected covered area (CCA) by the equations shown below.

$$
\begin{aligned}
& C A=\frac{N}{r \times c} \\
& C C A=\frac{N-r}{r c-r}
\end{aligned}
$$

In the formula, $\mathrm{N}$ stands for the sum of all enrolled studies (included overlapping) in all the SRMAs, while c and $\mathrm{r}$ represent the column and row products for the created study-citation matrix, respectively. CCA score $<5$ indicates mild overlap, scores between 5-15 suggest moderate overlap, and a CCA score $\geq 15$ suggests high overlap.

Secondly, the equation below will be used to evaluate the excess significance test (29).

$$
x^{2}=\frac{[O-E]^{2}}{E}+\frac{[O-E]^{2}}{n-E}
$$

Generally, the excess significance test is conducted to evaluate whether there is an excess of significant results for every article. On the other hand, the $x^{2}$ statistic will be used for testing the results of the excess significance test for SRMAs, and $<0.10$ is considered significant. $\mathrm{E}$ is expected studies or the sum of the power of the individual studies, $\mathrm{O}$ is the observed significant studies or the number of statistically significant studies in individual studies, and $\mathrm{n}$ is the total number of individual studies. An exploratory test for excess significance is provided in Figure 3.

\section{Discussion}

The present umbrella review will be conducted to 


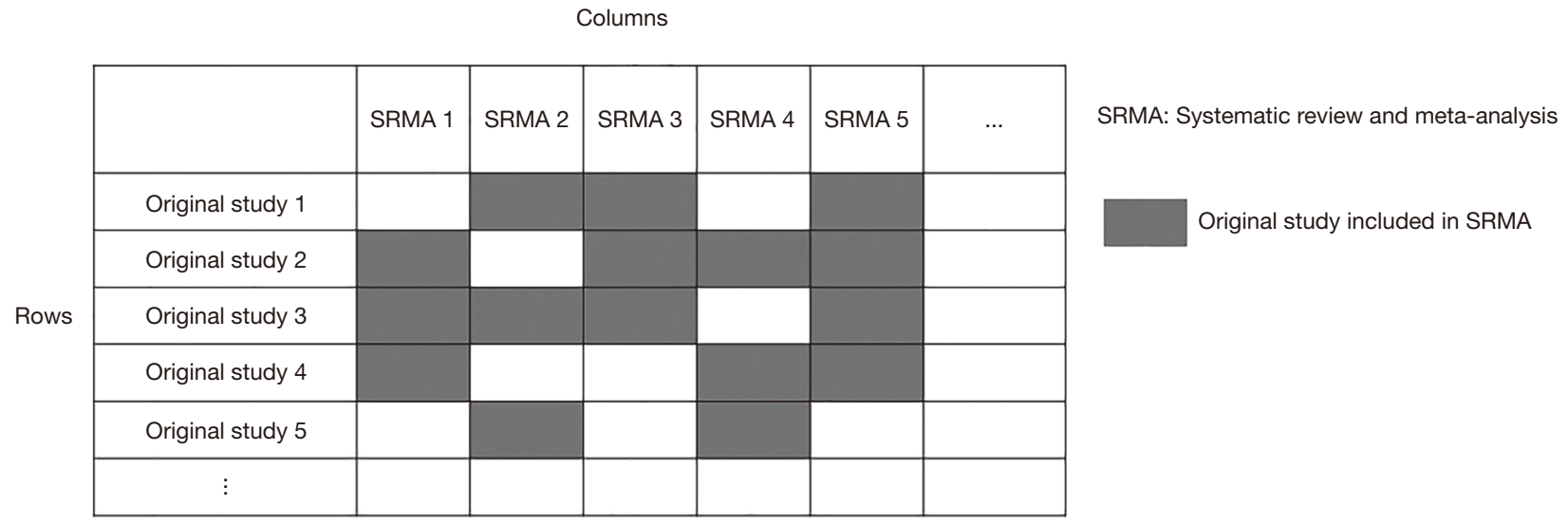

Figure 2 Citation matrix.

\begin{tabular}{|l|l|l|l|l|}
\hline \multicolumn{5}{|l|}{ Included systematic reviews and meta-analysis information } \\
\hline Title: \\
\hline Author/year: & Rate of control group & Effect size (RR, OR) & Total sample size & Power \\
\hline Original study author/year & & & & \\
\hline & & & & \\
\hline Total & & & & \\
\hline
\end{tabular}

Figure 3 Exploratory for excess significant.

synthesize the existing studies from SRMAs over the past 5 years. To this end, we will evaluate evidence quality, overlapping studies, as well as the excess significance test. Our review protocol includes the precisely defined question(s), criteria to include and exclude reviews, methodology of the critical appraisal for all enrolled reviews, the standard data collection procedure, and data presentation. Given the number of outcome indicators, multiple umbrella reviews may be published based on this protocol. An umbrella review is appropriate for evidence synthesis of $\mathrm{PR}$, as a range of interventions need to be investigated, existing research syntheses are available, and excessive evidence is urgently needed for informing novel procedures and policy (24). There are no clinical practice guidelines for PR in China; we aim to adapt existing guidelines on PR in ICUs to apply these in the Chinese context. Umbrella reviews will be a good supplement to the evidence of the guideline adaptation and to highlight whether the evidence is heterogeneous or not and determine the underlying causes of such results.

However, there are also several limitations to our study. Due to resource constraints, we will only search for English and Chinese SRMAs. Also, since we will only include
SRMAs in the past 5 years, there may be a loss of SRMAs published before 2016 .

\section{Acknowledgments}

Funding: The project is funded by the Science Research Foundation of Chinese Nursing Association (grant number: ZHKY201913) and Zhejiang University Academic Award for Outstanding Doctoral Candidates (grant number: 202059). The funders had no role in the design and conduct of the study; collection, management, analysis, and interpretation of the data; preparation, review, or approval of the manuscript; and decision to submit the manuscript for publication.

\section{Footnote}

Reporting Checklist: The authors have completed the PRISMA-P reporting checklist Available at http://dx.doi. org/10.21037/apm-21-626

Conflicts of Interest: All authors have completed the ICMJE 
uniform disclosure form (available at https://dx.doi. org/10.21037/apm-21-626). The authors have no conflicts of interest to declare.

Ethical Statement: The authors are accountable for all aspects of the work in ensuring that questions related to the accuracy or integrity of any part of the work are appropriately investigated and resolved. This study does not require ethical approval because individual patient data will not be included. There are no ethical concerns nor informed consent required.

Open Access Statement: This is an Open Access article distributed in accordance with the Creative Commons Attribution-NonCommercial-NoDerivs 4.0 International License (CC BY-NC-ND 4.0), which permits the noncommercial replication and distribution of the article with the strict proviso that no changes or edits are made and the original work is properly cited (including links to both the formal publication through the relevant DOI and the license). See: https://creativecommons.org/licenses/by-nc-nd/4.0/.

\section{References}

1. Bleijlevens MH, Wagner LM, Capezuti E, et al. Physical Restraints: Consensus of a Research Definition Using a Modified Delphi Technique. J Am Geriatr Soc 2016;64:2307-10.

2. Benbenbishty J, Adam S, Endacott R. Physical restraint use in intensive care units across Europe: The PRICE study. Intensive Crit Care Nurs 2010;26:241-5.

3. da Silva PS, Fonseca MC. Unplanned endotracheal extubations in the intensive care unit: systematic review, critical appraisal, and evidence-based recommendations. Anesth Analg 2012;114:1003-14.

4. Hofsø K, Coyer FM. Part 1. Chemical and physical restraints in the management of mechanically ventilated patients in the ICU: contributing factors. Intensive Crit Care Nurs 2007;23:249-55.

5. Tanios M, Epstein S, Grzeskowiak M, et al. Influence of sedation strategies on unplanned extubation in a mixed intensive care unit. Am J Crit Care 2014;23:306-314, 315.

6. Kirk AP, McGlinsey A, Beckett A, et al. Restraint Reduction, Restraint Elimination, and Best Practice: Role of the Clinical Nurse Specialist in Patient Safety. Clin Nurse Spec 2015;29:321-8.

7. Fink RM, Makic MB, Poteet AW, et al. The Ventilated Patient's Experience. Dimens Crit Care Nurs
2015;34:301-8

8. Kong EH, Choi H, Evans LK. Staff perceptions of barriers to physical restraint-reduction in long-term care: a metasynthesis. J Clin Nurs 2017;26:49-60.

9. Intensive Care Society. Guidance for the Use of Physical Restraints in UK Adult Intensive Care Units. 2021. Available online: https://www.ics.ac.uk/ICS/Pdfs/Physical_ Restraints_Guidance

10. Zhang C, Liu D, He Q. The characteristics of ICU physical restraint use and related influencing factors in China: a multi-center study. Ann Palliat Med 2021;10:1198-1206.

11. Pun BT, Balas MC, Barnes-Daly MA, et al. Caring for Critically Ill Patients with the ABCDEF Bundle: Results of the ICU Liberation Collaborative in Over 15,000 Adults. Crit Care Med 2019;47:3-14.

12. Perez D, Peters K, Wilkes L, Murphy G. Physical restraints in intensive care-An integrative review. Aust Crit Care 2019;32:165-74.

13. Gu T, Wang X, Deng N, et al. Investigating influencing factors of physical restraint use in China intensive care units: A prospective, cross-sectional, observational study. Aust Crit Care 2019;32:193-8.

14. American Psychiatric Association. Diagnostic and statistical manual of mental disorders: DSM-5. Arlington: American Psychiatric Association (US); 2013.

15. Kang J, Lee M, Ko H, et al. Effect of non-pharmacological interventions for the prevention of delirium in the intensive care unit: A systematic review and meta-analysis. J Crit Care 2018;48:372-84.

16. Lee PJ, Hahm BJ. Pharmacological Treatment for Delirium in Cancer Patients. J Korean Neuropsychiatr Assoc 2010;49:20-5.

17. Trogrlić $Z$, van der Jagt $M$, Bakker J, et al. A systematic review of implementation strategies for assessment, prevention, and management of ICU delirium and their effect on clinical outcomes. Crit Care 2015;19:157.

18. Deemer K, Zjadewicz K, Fiest K, et al. Effect of early cognitive interventions on delirium in critically ill patients: a systematic review. Can J Anesth 2020;67:1016-34.

19. Fuke R, Hifumi T, Kondo Y, et al. Early rehabilitation to prevent postintensive care syndrome in patients with critical illness: a systematic review and meta-analysis. BMJ Open 2018;8:e019998.

20. Cui N, Zhang Y, Liu Y, et al. Protocol for the adaptation of clinical practice guidelines for the management of physical restraints in critically ill patients. Ann Palliat Med 2021;10:4889-96. 
21. Harrison MB, van den Hoek J, Graham ID. CANImplement(C): Palnning For Best-Practice Implementation. Philadelphia: Wolters Kluwer (US); 2014.

22. Moher D, Shamseer L, Clarke M, et al. Preferred reporting items for systematic review and meta-analysis protocols (PRISMA-P) 2015 statement. Syst Rev 2015;4:1.

23. Shamseer L, Moher D, Clarke M, et al. Preferred reporting items for systematic review and meta-analysis protocols (PRISMA-P) 2015: elaboration and explanation. BMJ 2015;350:g7647.

24. Aromataris E, Fernandez R, Godfrey CM, et al. Summarizing systematic reviews: methodological development, conduct and reporting of an umbrella review approach. Int J Evid Based Healthc 2015;13:132-40.

25. Shea BJ, Reeves BC, Wells G, et al. AMSTAR 2: a critical appraisal tool for systematic reviews that include randomised or non-randomised studies of healthcare

Cite this article as: Cui N, Chen D, Zhang Y, Zhang H, He X, Ding C, Jin J. The effect of non-pharmacological interventions on physical restraint reduction in intensive care units: a protocol for an umbrella review of systematic reviews and meta-analysis. Ann Palliat Med 2021;10(6):6892-6899. doi: 10.21037/apm-21626 interventions, or both. BMJ 2017;358:j4008.

26. Zhang F, Shen A, Zeng $X$ et al. An Introduction to AMSTAR 2: a critical appraisal tool for systematic reviews. Chinese Journal of Evidence-Based Cardiovascular Medicine (Chinese) 2018;10:14-8.

27. Balshem H, Helfand M, Schünemann HJ, et al. GRADE guidelines: 3. Rating the quality of evidence. J Clin Epidemiol 2011;64:401-6.

28. Pieper D, Antoine SL, Mathes T, et al. Systematic review finds overlapping reviews were not mentioned in every other overview. J Clin Epidemiol 2014;67:368-75.

29. Ioannidis JP, Trikalinos TA. An exploratory test for an excess of significant findings. Clin Trials 2007;4:245-53.

(English Language Editors: C. Betlazar-Maseh and J. Chapnick) 